\title{
Genetic Diagnosis of Pachyonychia Congenita and Keratoderma
}

\author{
Frances J.D Smith ${ }^{1}$, Edel A. O'Toole ${ }^{2}$, C.David Hansen ${ }^{3}$, Philip Gard ${ }^{1}$, \\ Neil J. Wilson ${ }^{5}$, Eli Sprecher ${ }^{4}$, W.H.Irwin McLean ${ }^{1,5}$ \\ 1PC Project, University of Dundee, UK; ${ }^{2}$ Blizard Institute, Queen Mary University of London, UK; \\ ${ }^{3}$ University of Utah, USA; ${ }^{4}$ Tel Aviv Sourasky Medical Centre, Tel Aviv, Israel; \\ ${ }^{5}$ School of Life Sciences, University of Dundee, UK \\ University \\ of Dundee
}

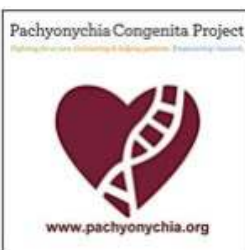

\section{Aim}

- To develop a registry of genetically confirmed pachyonychia congenita (PC) patients to facilitate clinical trials.

\section{Methods}

- The International Pachyonychia Congenita Research Registry (IPCRR) was established by the Pachyonychia Congenita Project to confirm diagnosis of pachyonychia congenita by genetic testing and to collate clinical and molecular data from PC patients worldwide (www.pachyonychia.org).

- IPCRR enrollment is by patient online self-referral or via clinician referral.

- Telephone consultations are performed by expert dermatologists combined with free genetic testing.

\section{Results}

- $P C$ is a rare autosomal dominant keratinising disorder.

- Characterised by severe plantar pain, palmoplantar keratoderma and variable hypertrophic nail dystrophy.

- Oral leukokeratosis, cysts, and follicular hyperkeratosis often occur.

Clinical features of PC

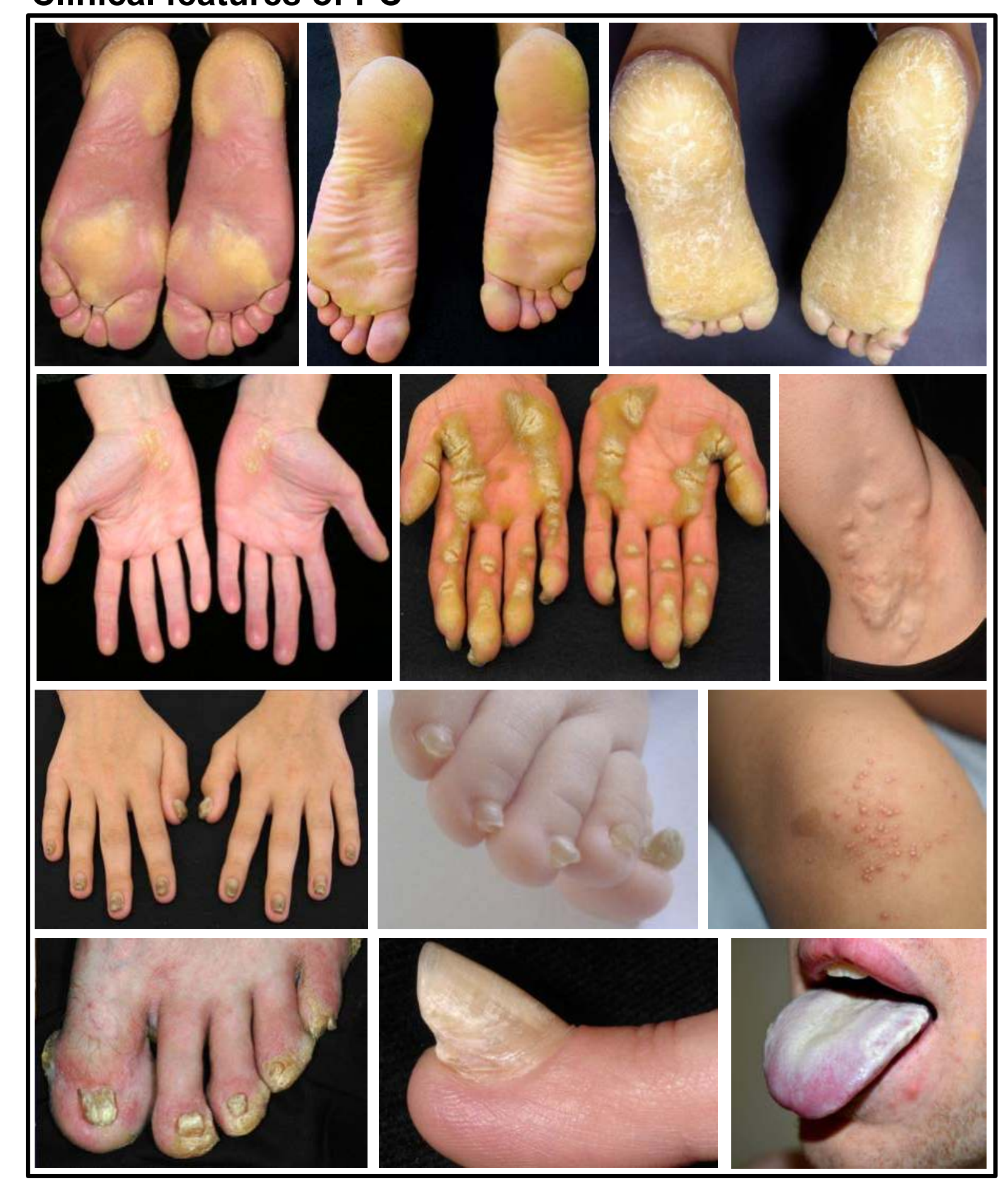

- The underlying genetic cause of PC is a mutation in any one of five keratin genes, KRT6A, KRT6B, KRT6C, KRT16 or KRT17.

- As of April 2017, the IPCRR had collected clinical and molecular data from 774 PC patients (419 families) worldwide.

- 113 distinct mutations have been identified.

- The majority are heterozygous missense mutations with a small number of insertion/deletion and splice site mutations.

Distribution of mutations in $\mathrm{PC}$ genes in 774 individuals (419 families) with genetically confirmed PC

\section{Results}

- An equally important service through the IPCRR has been exclusion of keratin mutations from a small number of misdiagnosed patients who present with some features of PC.

- In 52 cases we provided a correct diagnosis by identifying mutations (by Sanger sequencing) in genes including GJB6, TRPV3, DSG1, DSP, AAGAB and FZD6.

- With the development of Next Generation Sequencing, we developed a gene panel for the 5 PC keratin genes plus DSG1 (striate PPK), GJB6 (Cx30, Clouston syndrome), TRPV3 (Olmsted syndrome) and $A A G A B$ (punctate PPK).

- Mutations were identified in 46 of 51 cases recently enrolled in the IPCRR and screened using the panel. This included mutations in the 4 keratoderma disorders distinct from PC; KRT6A (21 cases), KRT6B (4), KRT16 (12), KRT17 (4), DSG1 (2), GJB6 (1), TRPV3 (1), AAGAB (1), no mutation (5).

- Four are previously unreported mutations; K6a p.Leu170His, K6a c.817-2A>G, DSG1 p.Trp303*, AAGAB p.Gly28Glufs*9.

\section{Clinical features of some other keratodermas}

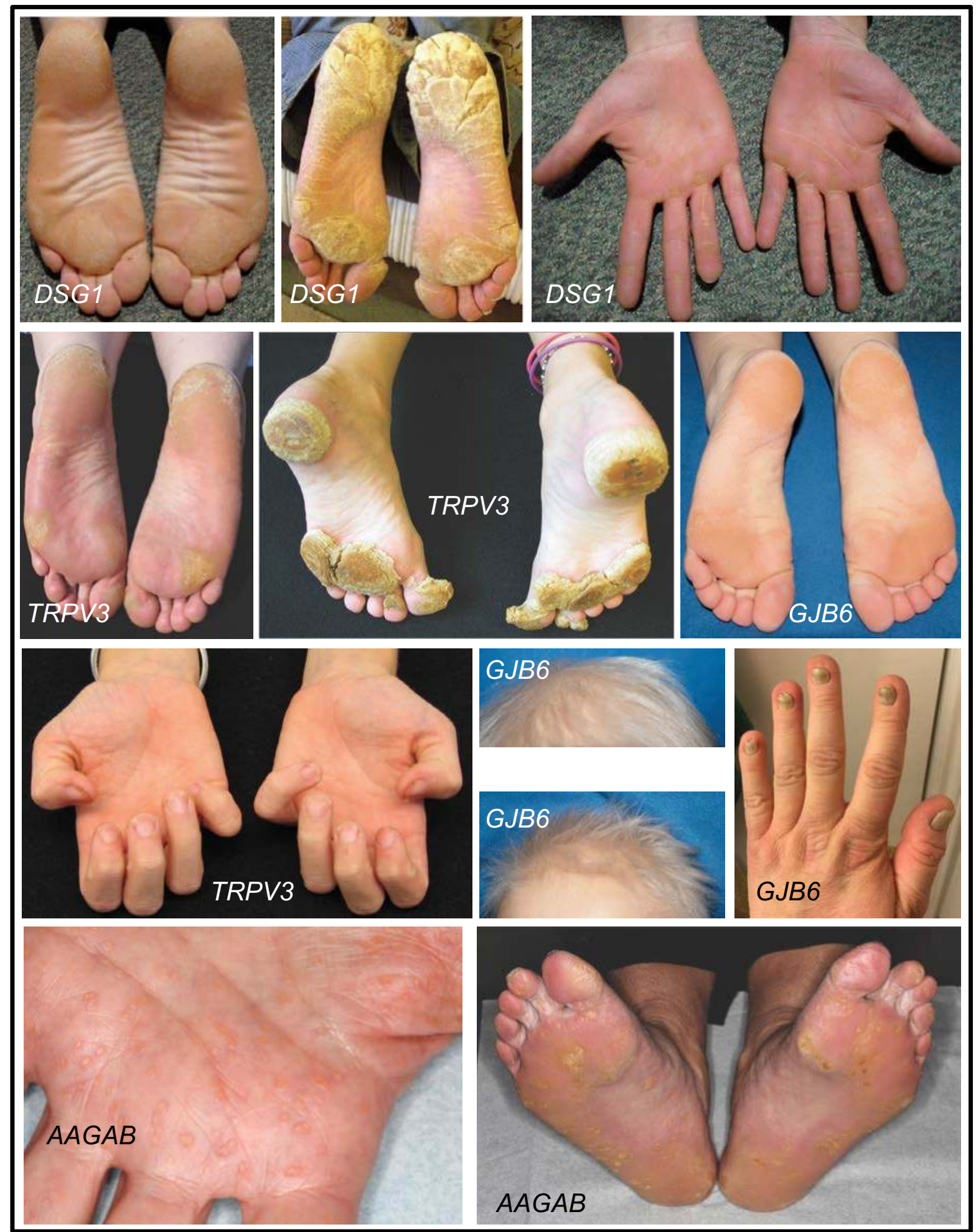

- The gene panel is being expanded to cover other known keratoderma genes. This will also help identify mutations in atypical cases or where Sanger sequencing failed to identify a mutation.

\section{Conclusion}

- Confirmation of a clinical diagnosis by genetic testing allows appropriate care and genetic counselling.

- Currently there are no effective treatments for PC and other keratoderma.

- The wealth of clinical and molecular data collected by the IPCRR not only ensures correct diagnosis for patients but is driving the development of future therapies.

- The IPCRR is a clinical trial ready registry of genetically confirmed patients.

\section{Acknowledgements}

Thanks to the many patients involved in the study. The work was funded by PC Project, 501(c)(3) U.S. Public Charity \& PC Project Europe (SC046480). 